\title{
A Framework to Abstract The Design Practices of e-Learning System Projects
}

\author{
Alain Corbiere \\ LIUM-IUT, Le Mans University, \\ 52, Route des Docteurs Calmette et Guérin, \\ 53020 Laval Cedex 9, France
}

\begin{abstract}
The use of ALT (Advanced Learning Technologies) creates dynamic sharing and exchanging between open source communities that diffuse elearning systems. In our opinion, the designer practices define new perspectives on e-learning design which are not structured and highlighted enough. This article shows the capabilities of a generic framework to analyse the design practices on a open source project and to explicit these practices. We describe how the semantics for architectural specifications proposed by RMODP (Reference Model-Open Distributed Process) framework were applied on an e-learning system project to analyse the principles of invariants, structural and functional.
\end{abstract}

\section{Introduction}

The current practices using ALT define new perspectives on e-learning design. First, the open source project applies a component-based software engineering [1] which makes it easier to specify and to reuse the software artefacts in design processes. Then, the e-learning designers are required to choose, understand and use the informational models provided by the IMS Global Learning Consortium to specify their decisions. The design intentions of an e-learning system are increasingly explicit. These facts bring us to search for an abstract framework for the engineering and the re-engineering of software system and to apply on an e-learning system. This framework must define a set of terms to explicit the observations on the practice of the open source communities that design e-learning platforms. This framework must be sufficiently precise to analyse and to describe the code and the architecture, and sufficiently open to allow its own interpretation.

In this article, we propose the RM-ODP (Reference Model-Open Distributed Process) framework which was adopted as an ISO (International Organization for Standardization) standard. This framework combines the logic of a formal description to specify the architecture and to consider the distributed information technology evolution [2]. Moreover, the objective of the design of e-learning systems is described as "a collaboration between the disciplines of educational science and computer science" [12]. 
This article illustrates the use of the framework by describing one interpretation of a four step specification on an open source project. We abstract the design practices of the e-learning system project and explicit the new perspectives based on the information model diffused by IMS Global Learning Consortium.

\section{RM-ODP framework}

This framework is defined as a meta-standard on the distributed system [3]. It is domain independent and supports a specification process. This tool allows us to describe the different design practices observed while respecting the vocabulary and to structure it according to its architecture. This framework provides two abstraction levels and it represents describes the transformations corresponding to the acts of specification, structuring and modelling.

The first abstraction level is defined by five viewpoints "each with an associated viewpoint language which expresses concepts and rules relevant to a particular area of concern in terms of which of a system can be described from that viewpoint" ([3] item 6.2.1).

- The enterprise viewpoint: which defines communities, actors, roles, activities and tools focused on rationales, opportunities and strategies.

- The information viewpoint: which defines the information handled by the various system resources and how it is dealt with by the various components.

- The computational viewpoint: which defines the software mechanisms that allow component diffusion and execution on software platforms.

- The engineering viewpoint: which focuses on the component mechanisms and functions.

- The technology viewpoint: which defines the material and software technologies.

The second abstraction level defined terms that allow us to specify the coherence between viewpoints. Four abstract concepts are used to specify our interpretation of e-learning platform project design practice:

- Decomposition: "To specify objects and behaviours which constitute an object or a behaviour" ([4], item 9.3);

- Template: "The specification of the common features of a collection of entities in sufficient detail that an entity can be instantiated using it" ([4], item 9.11);

- Behavioural compatibility: "An object is behaviourally compatible with a second object with respect to a set of criteria" ([4], item 9.4);

- Trace: "A record of an object's interactions, from its initial state to some other state" ([4], item 9.6).

These concepts help us to describe four specification tasks. These consist of the analysis the source code, the design framework and the technical specification models applied in an e-learning system project. 


\section{Instance of RM-ODP framework on an e-learning system project: OpenUSS}

By using the two abstraction levels proposed by RM-ODP framework, we explicit the design practices of OpenUSS ${ }^{1}$ (Open University Support System). This open source community manages a web site which allows access to the e-learning system and diffuses the source code be installed, executed, copied, distributed, analysed and modified. The stability and the continuity of this community, the variety of roles and the number of the users $(10,000$ users: students, professors and assistants) justify our choice.

We create an instance of RM-ODP framework on this project:

- To abstract the design practices on EJB (Enterprise JavaBeans) component technology in e-learning;

- To relate the informational models provided by the IMS consortium with an implementing entity ;

- To identify new perspectives of the ALT engineering.

\subsection{Step 1 : Using the decomposition concept to specify the computational entities}

This first step consists of specifying the platform's software architecture and describing the complex links between its components. We identify the first model published $^{2}$ on the OpenUSS web site. The dependence links use the UML (Unified Modelling Language) notation system describes the central position of the "foundation" component. This diagram corresponds to the first step of the abstraction process. However, this UML class diagram used describes the computational decomposition and the interactions between interfaces of different objects. This diagram corresponds to the computational viewpoint of RM-ODP framework. The analysis process must continue to transform the first specification into a more detailed specification. The technical system, on which these components are deployed, is a platform compatibility with the EJB component technology of Sun Microsystems. Each component is specified by the deployment models provided by OpenUSS community.

In addition, the decomposition logic applied by the technical framework of this platform identifies the object type "workflow" as being central. Its associated model describes the states of the computational interfaces. This allows us to observe the behaviour by tracking the methods invoked on the object interfaces, and the operations of the container object in which it functions. Focused on this viewpoint, the analysis is limited.

\footnotetext{
${ }^{1}$ See, http://openuss.sourceforge.net/

${ }^{2}$ See, http://openuss.cvs.sourceforge.net/openuss/openuss/design-general/model/src/
} 
This only communicates the production information to the technical administrator of platform. This information needs to be related to other aspects specified in the other viewpoints of the RM-ODP framework.

\subsection{Step 2 : Using the template concept to specify the engineering choices}

The decomposition of objects associated with the "foundation" component provided by OpenUSS community gives a list of objects from a computational viewpoint. The identification of the relations between two viewpoints allow us to transform a specification into a more detailed specification.

In this example, we look to model the relation which exists between the objects by specifying the computational objects from the engineering viewpoint.

The project OpenUSS designers apply a design process described in the technical framework EJOSA ${ }^{3}$ (Enterprise Java Open Source Application). It defines an easy to understand structure to be followed by the development with EJB components. This framework integrates a template which requires the naming rules for the source code files, and a structure in which to organize them.

Any designer who wants to collaborate with the OpenUSS community must respect this pattern when submitting a new component. The designer must save the structure of class in the "specification" file directory, produced by the analysis process. The UML class diagram used corresponds to the class saved in the "specification" file directory ${ }^{4}$ of "foundation" component. This UML diagram is the result of activity specification from an engineering viewpoint.

\subsection{Step 3 : Using the behavioural compatibility concept to specify the conformance points}

In addition, the RM-ODP framework defines itself as a guide to specify the conformance points between the ODP standard and a new specification. The act of specifying a compatibility between two objects bring us to define the relations between the specification selected and implementation the corresponding.

The conformance points depend on the terms of specification selected with observable behaviour in the e-learning platform. Moreover, for this framework the conformance points need to be identified and tests defined to satisfy in these points ([4], chapter 15). One of the four given conformance points classes defines "the behaviour of some physical medium so that information can be recorded on one system and

\footnotetext{
${ }^{3}$ See, http://www.enhydra.org/ejosa/

${ }^{4}$ See, http://openuss.cvs.sourceforge.net/openuss/openuss/dev-foundation/specification/src/
} 
then physically transferred, directly or indirectly, to be used on another system" ([4], item 15.3.4).

The IMS Learning Design informational model [5] is the physical medium chosen. It is produced by a normalization process which aims to propose models that answer to the interoperability needs between e-learning systems. In addition, this model is used in the design practice of e-learning platform for discussion on the pedagogical practices [7] and on the learning scenarios [8].

\subsection{Step 4 : Using the trace concept to specify the conformance tests}

This last step seeks to validate the behavioural compatibility specification. Our choice used the IMS Learning Design informational model to represent the trace generated by the method invocation of "foundation" component.

Three schemas provided by RM-ODP framework guide the specification activities from the informational viewpoint: the static schema, the dynamic schema and the invariable schema ([3], chapter 6).

By taking the static aspect of the XML (eXtensible Markup Language) schema of IMS Learning Design informational model as the invariable schema, our interpretation is to consider that the behaviour of the "role" entity is linked to the behaviour of "activity structure" entity and "environment" entity.

In addition, the dynamic aspect identifies new informational objects : "method", "play", "act", "role part", "condition" and "property". As specified in the IMS Learning Design specification document [5], these entities allow the description of the dynamic aspect of the scenario and correspond to the static schema from informational viewpoint.

\section{Assessment of this analysis: New perspectives of ALT engineering}

This article presents an instance of a framework on e-learning system project. We show the capabilities of the framework to support the designers team who wish to analyse the e-learning system design intentions.

The instance of the RM-ODP framework on the e-learning platform project demonstrates the four specification design steps. The effort of the OpenUSS community to communicate their e-learning platform design logics is highlighted in this example. The work of building relationships with new point of view proposed by another open source community is facilitated.

The aim is to understand and identify new perspectives on ALT engineering based on the abstraction of the design practices : 
- on educational re-engineering which applies the propositions of ALT. The IMS consortium questioned its capacity to describe the a priori behaviour early on. One of the new perspectives is to analyse the capabilities of a generic framework like RM-ODP to instrument specification activity. For example in [8], the authors define rules to guide the observable specification based on and to extend the XML schema of IMS Learning Design language;

- on software based components engineering where each component can be reused to design another and to support the negotiation between them [1]. The integration a software tracker, itself constrained by software architecture, is a complex task. With the RM-ODP framework, we can analyse the designer practices on the OpenCPMS (open Controlling Performance Measurement System) component. It's a proposition of a tracking manager of the OpenUSS platform.

- on service engineering [11] which look to mapping the notion of business process and the business process life cycle onto learning processes and the learning process life cycle. This perspective structures and specifies the politics and the collective behaviours of a "community" defined in the business viewpoint [13]. These specifications will facilitate the exchanges between e-learning system projects;

- on model driven engineering where the difficulty is to identify the mechanisms used to model the various software artefacts [9]. The open source community OpenUSS [10] proposed one of the first contributions in the e-learning domain. One of the new perspectives is to detail this proposition by using RM-ODP framework concepts and viewpoints.

One of the stakes of the e-learning designers' open source community is to have a framework such as RM-ODP to propose a common vocabulary to share their practices and to identify new engineering and re-engineering perspectives to accommodate advances in ALT.

\section{References}

1. Szyperski. C (2003) Component Technology - What, Where, and How ? (USA). doi:10.1109/ICSE.2003.1201255

2. O'Rourke C, Fishman N; Selkow W (2003) Enterprise architecture using the Zachman framework. THOMSON LEARNING, Boston

3. ISO/IEC-10746-1 (1998) Open Distributed Processing Reference Model, Part 1: Overview. 1SOIIEC JTC1 SC7

4. ISOIIEC-10746-2 (1996) Open Distributed Processing Reference Model, Part 2: Foundations. ISOIIEC JTC1 SC7

5. Koper R, Olivier B, Anderson T.(2003) IMS Learning Design Information Model, version 1.0 Final Specification.IMS Global Learning Consortium

6. Barré V, Choquet C (2005) Language Independent Rules for Suggesting and Formalizing Observed Uses In a Pedagogical Reengineering Context. doi: 10.1109/ICALT.2005.189

7. Burgos D, Koper R.(2005) Practical pedagogical uses of IMS Learning Deslgn's Level B. SIGOSSEE Conference. http://dspace.ou.nl/handle/1820/471. Accessed 01 April 2008 
8. Pemin J.-P., Lejeune A. (2006) Models for the re-use of learning scenarios. http://dspace.ou.nl/handle/1820/580. Accessed 01 April 2008

9. Bézivin J.(2006) Model Driven Engineering: An Emerging Technical Space. doi: $10.1007 / 11877028$

10. Grob H. L, Bensberg F., Dewanto B. L (2005) Model Driven Architecture (MDA): Integration and Model Reuse for Open Source eLeaming Platforms. Eleed Journal. http://eleed.campussource.de/archive/1/81/. Accessed 01 April 2008

11. Helle D, Hrastnlk J, Maurer H (2005) An Analysis of Application of Business Process Management Technology in E-Learning Systems. Proceedings of E-Leam 2005 MCE Charlottesville USA $2937-2942$

12. Cees V, C Jones V, van Sinderen et al (1997). Tele-education process modeling supported by the ODP viewpoint enterprise language. doi:10.1109/EDOC.1997.628341

13. ISOIIEC-15414 (2006) Open Distributed Processing Reference Model, Enterprise language. ISOIIEC JTC1 SC7 